\title{
PROBLEMAS TENDMOSOS DE LA MANO Y SU TRATAMIENTO
}

\author{
DR. TUUMBERTO RAFOS:
}

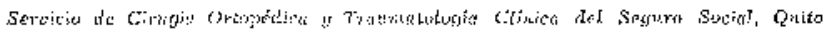

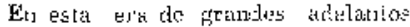
cientíliess, cuantlo :s practica con éxi-

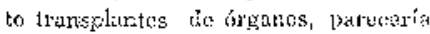

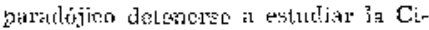
ruşia de lil Mauur, ryue ha estedo enco.

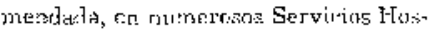

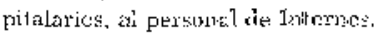

Al inuditar cremos clue esos atelä.

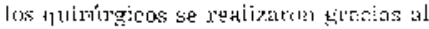

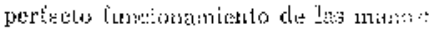
sin ollas; no exirtiriu progrtemb, sun tie-

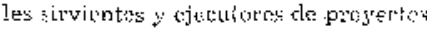

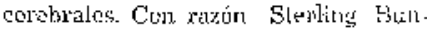
nol", hombre pensector y te accisin da pusc en su respectivo sitid, a] rlec: "1 IA MANO, LUWGO DEL CERE,BRO, ES FL MEFOR DF LOS IIEPES IUL IIOUIR,RE".

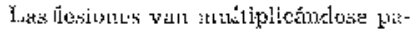
ralelumente al prosrese ka let izdustriułzación. LLs traumatismos de trenno están en proporéón mayor al de conjututo corpural, atribuible al trabajo manual caractrristico de la especie bu. mana y ata la acción de protección.
En da Clénicu del Sengur de Quito. en atconcionas $y$ un 42 ir de las lesiones tranmáttes rortesponden a mano?

ras estadístieas demuestran que la:

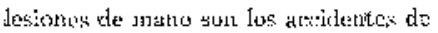
triotijo más frecuentos, fuo demandan

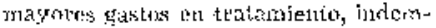
nizistion y pensionex. I da sinuatión hat empecirads (en los viltimos años, debids a la accleración, indusirializactiólt, moécanización del trahajo y accidentes rle

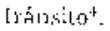

In clatos gublisados por ta oficina de Riexgos dal T'rahaju del Drportamento Médico del Snguro Sociä en el año de 19tifi, en la Provirusia de Pieti:n1" chat, se hum registrutho 384 accidentes ide latbajo, de los cuales 1.17 tortespordens a mitho, o sea, ten $36 \%$. L cos. to total por indemnizaciones asciende a I'000.000 de sucres, curresprmdiendo a la mano aproximadimente 360.000 . Ester elemuestri la impertancia die prenetuparnos dei pmolicma". 
Jin la mano todos los elementos ws. ingportantíes: cubierta sulánca, icudones, nervins, sistema arterio-venusil, huesos y articulaciorses, se compl: mentian $y$ si hay falla de uno de elto. va ten mengua del correcto furcionsmicnto.

Entre las lesiones vá́s impnotlantes $y$ «que son cibjeto del presisute trabajo, teremes as correspondenles a los tend():es, partes motoxis drel compi:-

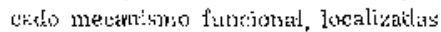
em fiexorcs, extensores y en ol apira(i) extennes det Jos dedtes, estac últims. cumplejas y de por's frerumeia.

\section{MATERIALTS Y METODOS}

Sc han Homado 50 ensos atendidess on la Clíryica tel Seguro, di: Quito, tanto cr el Seuvicio Hospitalario como en Comsulta Exxterna,

Lin la ristadistica encontramos: qu eI $84 \%$ corresperden al sexo mas:uJi110 ei lof\% al femmins; es raxisn de que los trabajos matsuales en la indusidria están enconendatsos ol sexor mas. sulinrs.

Fn cuinto h edad en ] d cua] cotos accidentes se producen, ta dhécada de 30 años $e s$ li de mayor frectuencia $40 \%$, siguieryda la do $40,20,50$ y 60 años en su orifen.

El mecarimo eausal cic mayor: incim demeji comesponde a los tritumettismos t:errudos, sigaienuo üus loeridas por vidric, srawhacamientos y urz caso pox

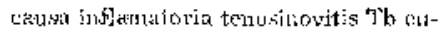
bit ull.

Iia aicneión definitiva se realizá on 22 pacientes a los varios diess de la le- sirin; ra ]8, a !rs pocos reces; an 7 des.

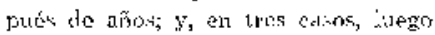
det pocas horas.

$\mathrm{E}]$ mayor No de cascs sue lratadu en Servirios de Emergumeja con Cirigía clifelida.

Cortrespunden 26 asos $52 \%$ a $6 \mathrm{fl}^{3}-$ xorca y 24 (18\%) a rxtwintres, l*)ntabiL:zendu entre cotos, it arrespanilientess a issones et bandelctas laterales del apacuta extemsor tle las dreclos.

Do los flexores 1 corresponde al $\mathrm{I}_{1} \mathrm{l}$ l15ar, 6 al findice, 5 al mett:o, 2 ul isnul:ur, 5 ai meñiquc, $y$ lesínes mít.iples 7. El julgar es el jucros afnetado debido

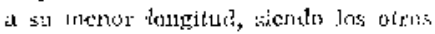
los unás explicstos a dus tlaumatisinás (realizan la pichsión simese).

Lá zona "tierra de naclico", es donder asientar con navor frecuen:ia las lesiones (11), siguiendo lin zona I (3), pà̀m de mans 5, Ju fulinge distal y' lung: carpianc $4, y$ munce 2 .

En los extenismes 6 sirresponcien at meñitue, 4 al rnedio y aumlar, 2 al puIgar, Worso de xhane, y a taros con lesiones rutultiples.

Eu 1.2 tésos $24 \%$ coexistion Jesión

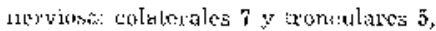
(2 e:1 cubital, 2 cn medians y 1 en mom clingls $y$ cibitati.).

Fo liss intervenciones yuirúrgicas sto

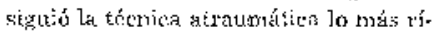

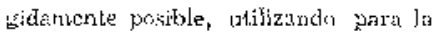

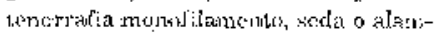
bre cut puscas casos. Fis. injertur tendiansos fute e? palmes menus el dousante.

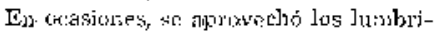

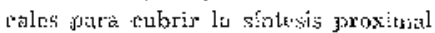

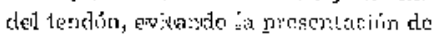
problemas uiterieres?. 
Soure liexulus at pratiatalm 9 irsjertos temdinosos (con tenadesis clel ca-

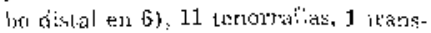

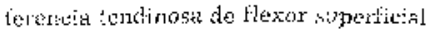
del ancolua a flexer profurdo del xenique es el taso de teturinovitis "'b: cort destruecion itudinosiar a nivel fle paloma de gramo.

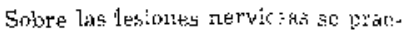
ticarun 2 13eturitisis de colateritles, 10

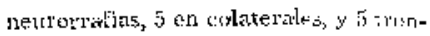
culares.

Se rointervinicron a casos pur unalos resultudos: 1. Lexchisis, l acorlatouento Lendinesso $y$ lernligis y 1 injerto de piel por teerestis du la nisirut.

\section{R.HSLTATAOS:}

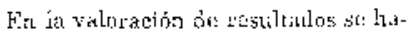

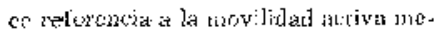

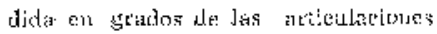

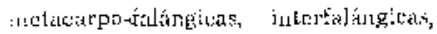
j: stuma (areu the muvlióad). La clistan cia en ceistínterirs de pulpojo a dilliege clistak palmus.

La ltilitdad rue prosta el declo, si realisal la prensión nenor $y$ la mavor con suîicicnte fuedza. I a strusibilidach existorie y si conbtituge wa dedo problemá. La cosméiléci.

De axuerdo cor: cstos pal'ámetros los resultartos oblenidos licgo de las in-

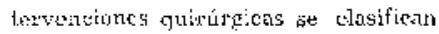
en luenos, regulares y uratos, Tubla I.

\section{TABLA I}

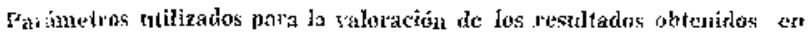
correcciones quiríxugicas de lesiones de mano

\begin{tabular}{|c|c|c|c|}
\hline & FL'FNO & EHX:LLAR & $3 \mathrm{TIBO}$ \\
\hline Angulie muvijiclut & $\overline{170}---$ & $\left.\right|_{1} ^{-} \cdot{ }_{11 j^{\prime}} \cdot-$ & $11.5^{2}$ \\
\hline Dist:nusikt j, r. & 2,5 sill. & 5 เn. & ถ̀ cas. \\
\hline Fr:vaión maya: & C: filiziza & guticisote itse:zz: & צ:al \\
\hline Sensitiil:dal & Breni. & AlEeנada & Aǚnte \\
\hline Deis: prolilased & Xis & $\begin{array}{l}\text { Pars cicrlos } \\
\text { movitniensas }\end{array}$ & 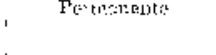 \\
\hline Costulika & TH11:!ו! & Ac:1:!table & Nit:: \\
\hline
\end{tabular}

\section{Resultaulus Bustos:}

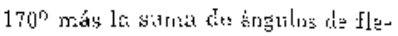

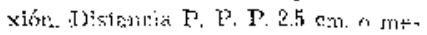
nius.

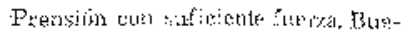
na semsibiliducl. No cinctitupe 3 ho

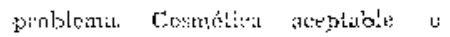
bitejas.

\section{Jarinlitados Rebjulates:}

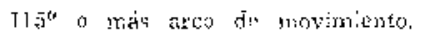
Distancia E. F. P. 5 cll.. o metar. 


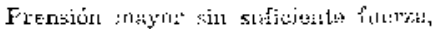

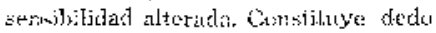
problema para ciertos novimientos. Custnélitaturendt? areptable.

\section{kesultados thalos:}

$115^{\circ} 0$. mentes areo de movimiento.

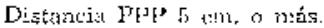

Pletsión mestor prestate? 0 ind hersic.

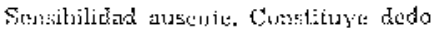

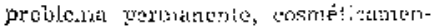
te miter.

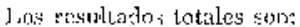

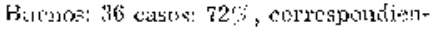
du a los flexnres 1!; ; imsores $15: 34 \%$

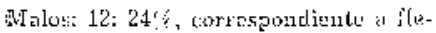
xores 5: 1nc, extensores 7 : 14\% Cazos en estudin 2: (4\%) olic currespenders a flexores.

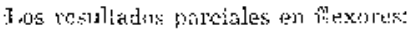
Ii:jertos 96 bues. 33 en estutio $2 \mathrm{y}$ l mo.o, tanorefinis. 11 (8 buenes y 1 res $x+2]_{(0 x)}$

Solore los cxt+matores se practicuron:

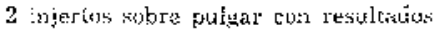

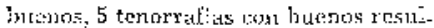
tados, tonodexis de calo rlistal do exteman 12 , wor: 6 resultados buenos $y \quad 6$

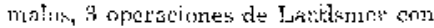
2 resultitla; bisaros y uno nuald.

\section{) HISUSION}

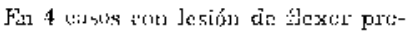
fermdo sil: tratamiento quirúroico, ls funcion eral aceptable, no asi cutando sc exploró pur liscíjiz de f́,exor profiundo нл жия 2 con integridnd \&t: superij-

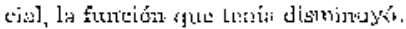

Cirnur cumplicacienes so proentaron:

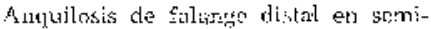

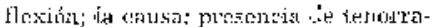

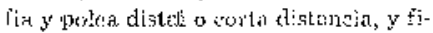
țación de las ntistratis o lesión de las fibras oblicuas dich retina[n] $]_{12}$ ? Cuando se pracícó temutess dc: cabo distel, ste

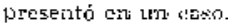

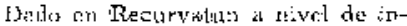

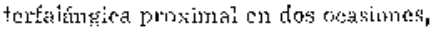

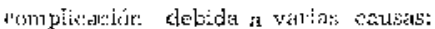

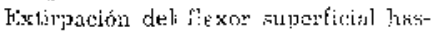
tin su insereión ósen, sin drjar dats banthlowas deterales; mala posicione de la

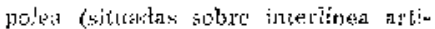

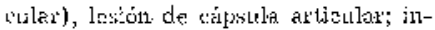
ferto tentinoso demasiendo lexgo, lesín ke bandcictas latt:ratem del apitrato cxtensor $y$ lisamerto retinatuar

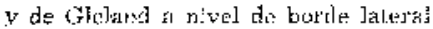
sligutal, canbiazidy la direcrión de lis fuerya de palanca qut: lealiza ta bandesenta laterul ert ayurta a la flexión de

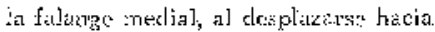

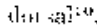

Rozón prara yue ?a jucisión y disec-

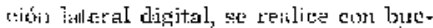
na planticasion despetands en lo posi-

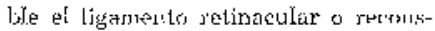
truyendolo al lesionarte. Rendizamos

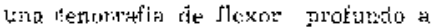
1:

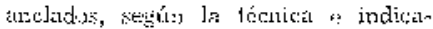
clcres Natas por Clavde Vertan. ${ }^{12}$ con rial resultado; pn Ia 1 t:tizìa a los 4 mesces, so constata adserers:tas a planos protulodos y stiperficistes, destlesamiento doil filexor pro- 
fundo an ripgull de falange proximal. Jistu lícrivat en munos axpertas dit

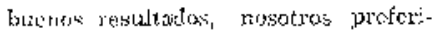
Irus a este rivel, injerto iendinoso,

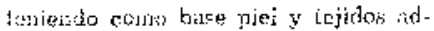

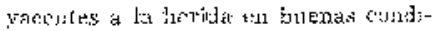

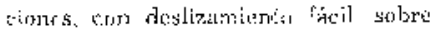
1)

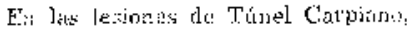
liis tenorrafiats de tifexoles pyrofundos tar buerjos reibliados. La recuperitción es todal en ins Nepurrafias de

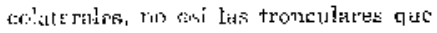
M.jeran lá sconsibilixtud, jem mom le: inutilitad.

Rivantatuos un raso de sención de exicusor largo del pulgur comon eom-

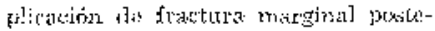
rinii cte ratio y otra de scecínt de cxtedsuress largo y corto klet pulgar port horida vertands, cratadas con injexto

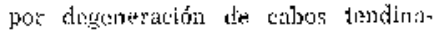

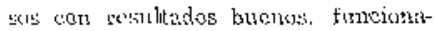
enicnict reculuerado en zran porrestaje $y$ carto titemps.

Lis tenoderis diemon $50 \%$ de rosidfrados buenos y $50 \%$ de malos, guizé debido a marlial técuies, disección insuldicjentr do crbon terdinoso, mala im-

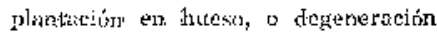
ionizjnosa revinzarixlo la fijasión do tejido fibroso reemplazanté.

Fan interventiones clectuadias en pha-

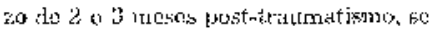
obtuvieris bllesnis resulteudos.

Se lrataron toes lociones rlo buladedetas laterailes disl apirato extenisor: madhacamiento del desto medio er: rodilo, incapaciturido complctaments la fexiótu, com impresión diugnóslicu de cesión de floxures; en tol noto operatertio, hubo inlogritiad de: Itexures. Se roaliza disecóth y trasposición de bandcicto bateral de $i x l e n g o r$ hacía zona palmins consorvitrato du iuserción distal, $y$ lorumando pronte el liginnento de C!tlinste" (opetarciot:l de Tuansthror), rem bucinos resuTintos.

lil seguntin casts, luego dc inmovili-

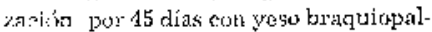
ma kis una fractura de trapecio, al exa|nes se encontró que la cxtensión com-

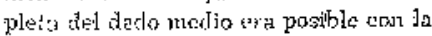

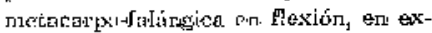
tensirin habia hiperflexión de falarge distal, couj ligmo reciuvatur, dando la impresinin die rontractura de intrínse-

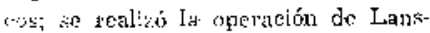

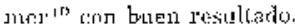

Ell al terror rylso tratedo anterior-

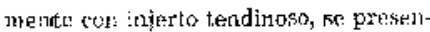
16 recurvatun y nisssutia flexión, que jnicitbe ni modificul positivamenle el rutulvatum. Con la eperación do Landsmer amplió la flexión a $\mathbf{1 0}^{\circ}$, el mrobtema fenia onra causa, se reintesyino para cfochuar und tennlisis ulurtamienio de torechón, di caso está elu recuperación, pero se cbscerva mejorík, hay movíruinnir de flexión.

\section{RESUUHEN}

Se estudiarrm 50 casos, de lessiones tendisosnis dis mo stuadas at nivel di los fitexores in extensores y aljacialo extensor do los dedna. Fr herirlas limpias $y$ recientes o fuera de la "fierma do nudle" rusfins primarins. Los injertos tendinowos se realizaron en casos diferides. 
lil área de piel bujo suyo nivel se prêticó ca plastia tordinon, dobió estar isbre de tejicto cicesturizetl o libroso,

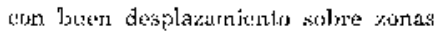
protundas, para evitar procesos adhen rerctiales.

J.a rextlitisurión sio ]a Josión se hizo. lo más pronto posidzle, evitando ta jro-

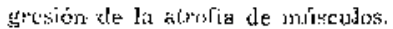

T. dippitales se pland:curon con buena téniar il in devitar letiones due com.

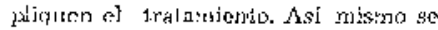

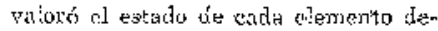

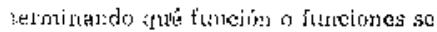
podráu drevalucr, buscondo las més ne sesclitia de acuetdrs al irabajo que de. ucu:prña.

\section{SUMNATRY}

Fif $\mathrm{f}_{\mathrm{y}}(\mathrm{5} 0)$ ceses of temdinums lesions of the haricl at the level of flexores and texteusors and extensur appirrathus of the fingers wero studicd. It cloan and regent wound primary tenorraphian

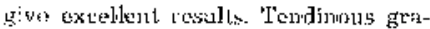
fts were rea]ized in differed oases.

The arca of gkin below which the

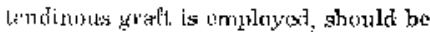
free of scurrec or tibrous tiasule, with gscod displacement aroturd the deep zohes so thes adthesionls will be avoided.

The herling of the lessions was ascomplisined as sorm as yossible, avoidings progression ot museulat aitrophy. Tho intervent on the incisjous and dissections of the latertal fingers was pling. ned with the end of aroiding Iesions that complisente teratment. Thus, the ztote of each elemenl. was tuterminet for its value is recurning the function or functions, Jooking for the most ne- cessary ones accordinot to the work to be carries ort.

\section{BIBLIOCHAFIA}

( RLNNEL: Cirugia de la Nano intermé

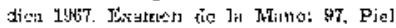

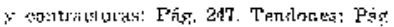

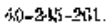

3 wNIly MsR'CIN: Skerling Hungath on Gitunte suirúlyticu. Clenicas quirutegicos de Forle Amúvicn, Agosto de 1081, Pain. \&

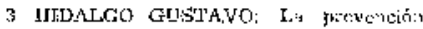

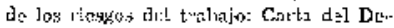

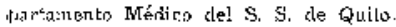
1367.

4 JEKKin Fa: Lesiones intamáticas de M:ıлo: Gacela Sanitaria, México 20, 1965 J'도․ 13.

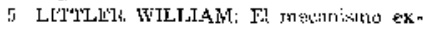
tensor del decio. Clinjeas Quirúngetes de Nojle Anếr:ca, Abri! 1967, Fág. 41.s.

¿i IJNDSSAY: Reparación rluneda del tunm tow flexor de los dedos, Plastir ariel ken const.ruative Surgery, V.0!. z 23, NQ $h_{1}$ Docominer 165jol. Hás. 6l2.

7 RANlOS HEHBERTO: Frulícma CiáTeo en trautnatismos de Marto, I Jorisadas de Ortapedia y Tramsatologta, Quiโo, 1967.

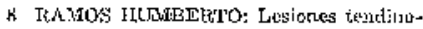
sas de Mund: 1966. Instifuto Mexieano del S. S. Contro Mécico Nacionat. Hosp. do Trumuatología.

9 SILWLINAFIO SOLFE CIRUGIA DF LA MAYO: Husp, de Thumatologia. Centro Móding Kaciorel. E. M. S. S.

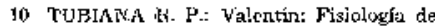
la cxiensión de lus dotos: Clínicas Qui-

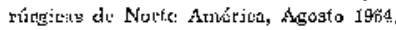
Pạc. Aot.

11 TORRES IOTRGE: Problunwa cutáthen en t'inumatiexo de iz Mann: Tesis Doctoral: 11\%ö8, l’álg. 2.

12 VFIDAX CLADE: Cousiderinciones

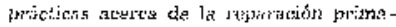
ria s seculdarid en las lesiones de Jus twadons r.uxores. Ulínicas Quirúrgicas the Nortc Aneirica: Agosto 1964, Päg. 851 . 